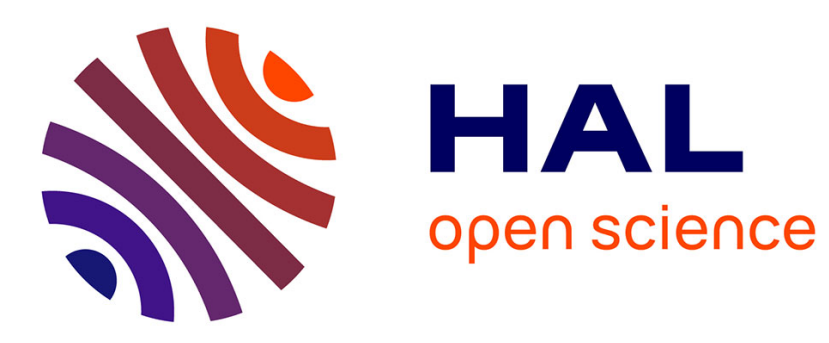

\title{
Finite memory observer for switching systems: Application to diagnosis
}

\author{
Abdelfettah Hocine, Didier Maquin, José Ragot
}

\section{To cite this version:}

Abdelfettah Hocine, Didier Maquin, José Ragot. Finite memory observer for switching systems: Application to diagnosis. 16th IFAC World Congress, Jul 2005, Pragues, Czech Republic. pp.CDROM. hal-00151264

\section{HAL Id: hal-00151264 https://hal.science/hal-00151264}

Submitted on 2 Jun 2007

HAL is a multi-disciplinary open access archive for the deposit and dissemination of scientific research documents, whether they are published or not. The documents may come from teaching and research institutions in France or abroad, or from public or private research centers.
L'archive ouverte pluridisciplinaire HAL, est destinée au dépôt et à la diffusion de documents scientifiques de niveau recherche, publiés ou non, émanant des établissements d'enseignement et de recherche français ou étrangers, des laboratoires publics ou privés. 


\title{
FINITE MEMORY OBSERVER FOR SWITCHING SYSTEMS: APPLICATION TO DIAGNOSIS
}

\author{
Abdelfettah Hocine, Didier Maquin, José Ragot \\ Centre de Recherche en Automatique de Nancy \\ Institut National Polytechnique de Lorraine \\ 2, Avenue de la Forêt de Haye \\ 54516 Vandoeuvre les Nancy Cedex, France \\ \{ahocine, dmaquin, jragot\}@ensem.inpl-nancy.fr
}

\begin{abstract}
In this paper, a fault detection method is developed for switching dynamic systems with unknown inputs. These systems are represented by several linear models, each of them being associated to a particular operating mode. The proposed method is based on Finite Memory Observers and mode probabilities with the aim of finding the system operating mode and estimating the unknown input. The method also uses a priori knowledge information about the mode transition probabilities represented by a Markov chain. The proposed algorithm is of supervised nature where the faults to be detected are a priori indexed and modelled. In this work, the method is applied for the fault detection of a linear system characterized by a model of normal operating mode and several fault models. Then, it applied for fault detection in the case of a linear system with unknown input where state and unknown input estimation are done simultaneously. A comparison with the Generalized Pseudo-Bayesian method shows the validity and some advantages of the suggested method.

Copyright ${ }^{\circledR} 2005$ IFAC.
\end{abstract}

Keywords: Diagnosis, switching system, multiple model, finite memory observer, unknown input, state estimation.

\section{INTRODUCTION}

As an evidence, control of systems is becoming more and more sophisticated; that is due to the combined fact that systems are naturally complex but also because it is often desired to manage all things affecting the system. This motivates researches on reliability, availability and security. In this field, FDI (Fault Detection and Isolation) has been developed over the two last decades (Patton et al., 1989), (Blanke et al., 2003). A common way to FDI is often based on the state estimation of a process which also produces an estimation of the process output. In general a simple comparison of estimated and measured outputs is used to design a set of residuals that are sensitive to faults. Thus, state estimation is a key point of FDI. Generalized Pseudo-Bayesian first order approach (GPB1) (Bar-Shalom, 1990) is a powerful tool to track the behaviour evolution of the process, with also based on residual information. In fact, process can be characterised by one or several models for normal operating conditions and by another set of models describing the different situations of misfunctionning affecting sensors and actuators (that are 
the consequence of damage of process components). This set of models can be used to describe the overall behaviour of the process. For this purposes, the multiple model strategy which is often used is exploited in the GPB1 approach. In the topic of state estimation, the GPB have a wide range of applications mainely in tracking targets (Bar-Shalom et al., 1989),(Bar-Shalom, 1990). In the last few years, the multiple model approach has found a wide spectrum of application, including state estimation (Bar-Shalom et al., 1989), (Bar-Shalom, 1990), (BarShalom and Li, 1993), (Hanlon and Maybeck, 1998), control (Murray-Smith and Johansen, 1997) and modelling (Gasso et al., 2001). In general, a parallel bank of filters is used; where each filter is based on a local model representing a particular behaviour of the real process. The evaluation of the residuals between filter outputs and observed process outputs allows one to design fault detectors. The GPB method is based mainely on the Kalman filter and mode probabilities. In this work, the Kalman filter is replaced by a Finite Memory Observer (FMO) (Kratz et al., 1994),(Medvedev, 1996),(Nuninger et al., 1998) which shows interesting characteristics that are mainely related to the fact that the estimate at time $k$ is independent from previous one at time $k-1$. Moreover, a FMO is less influenced by system noises compared to the Kalman filter.

This paper is organized as follows: In section 2, the development of the finite memory observer is described. In the section 3, the FMO is used for the estimation of the unknown input. The section 4, presents the proposed method based on an FMO, within the framework of switching systems, in order to detect the changing regime. In section 5 , the FMO with unknown input is used for simultaneously detecting the changes of modes and estimating the unknown input. Finally, a conclusion is drawn on the use of FMO for switching systems and on the suggested techniques.

\section{A FINITE MEMORY OBSERVER}

A Finite Memory Observer uses measurements in a finite time interval only. Consider the discrete time and invariant following system:

$$
\left\{\begin{aligned}
x_{k+1} & =A x_{k}+B u_{k}+G w_{k} \\
y_{k} & =C x_{k}+v_{k}
\end{aligned}\right.
$$

where $x_{k}$ is the state vector at time $k, A$ is the state matrix, $u_{k}$ is the input vector at time $k, B$ is the input gain matrix, $C$ is the output gain matrix, $v_{k}$ and $w_{k}$ represents respectively, the state and measurement noises and $y_{k}$ is the output of the system at time $k$.
In the noise-free case, the system is described by:

$$
\left\{\begin{aligned}
x_{k+1} & =A x_{k}+B u_{k} \\
y_{k} & =C x_{k}
\end{aligned}\right.
$$

Observing the system evolution on the time horizon $[k-m, k]$ and following the classical Chow-Willsky scheme ((Chow and Willsky, 1984)), we can write:

$$
Y_{k}=P_{m} x_{k-m}+B_{m} U_{k}+G_{m} W_{k}+V_{k}
$$

with :

$$
\begin{aligned}
& Z_{k}=\left[\begin{array}{llll}
z_{k-m}^{T} & z_{k-m+1}^{T} & \ldots & z_{k}
\end{array}\right]^{T}, Z \in\{Y, U, W, V\} \\
& P_{m}=\left[\begin{array}{llll}
C^{T} & (C A)^{T} & \ldots & \left(C A^{m}\right)^{T}
\end{array}\right]^{T} \\
& B_{m}=\left[\begin{array}{ccccc}
0 & 0 & \ldots & \ldots & 0 \\
C B & 0 & \ddots & \ddots & 0 \\
C A B & C B & \ddots & \ddots & \vdots \\
\vdots & \vdots & \ddots & \ddots & 0 \\
C A^{m-1} B & C A^{m-2} B & \ldots & C B & 0
\end{array}\right] \\
& G_{m}=\left[\begin{array}{ccccc}
0 & 0 & \ldots & \ldots & 0 \\
C G & 0 & \ddots & \ddots & 0 \\
C A G & C G & \ddots & \ddots & \vdots \\
\vdots & \vdots & \ddots & \ddots & 0 \\
C A^{m-1} G & C A^{m-2} G & \ldots & C G & 0
\end{array}\right]
\end{aligned}
$$

The estimate of the state $\hat{x}_{k-m}$, at time $k-m$, which minimizes the the criterion $J_{k}=\| P_{m} x_{k-m}+B_{m} U_{k}-$ $Y_{k} \|^{2}$ subject to $x_{k-m}$, can be obtained easily using the least square method

$$
\hat{x}_{k-m}=\left(P_{m}^{T} P_{m}\right)^{-1} P_{m}^{T}\left(Y_{k}-B_{m} U_{k}\right)
$$

The state estimate at the final time $k$ of the observation window is obtained by integrating the system (2):

$$
\hat{x}_{k}=A^{m} \hat{x}_{k-m}+T_{m} U_{k}
$$

where

$$
T_{m}=\left[\begin{array}{lllll}
\left(A^{m-1} B\right)^{T} & \left(A^{m-2} B\right)^{T} & \ldots & B^{T} & 0
\end{array}\right]^{T}
$$

Thus, at each time $k$, the expression of the state estimate is defined by:

$$
\hat{x}_{k}=R_{m} Y_{k}+S_{m} U_{k}
$$

with

$$
\begin{aligned}
R_{m} & =A^{m}\left(P_{m}^{T} P_{m}\right)^{-1} P_{m}^{T} \\
S_{m} & =T_{m}-A^{m}\left(P_{m}^{T} P_{m}\right)^{-1} P_{m}^{T} B_{m}
\end{aligned}
$$

\section{A FINITE MEMORY OBSERVER WITH UNKNOWN INPUT}

The finite memory observer can be used for the estimation of unknown input by considering it as 
a state variable of the system. The unknown input system is written as follows:

$$
\left\{\begin{aligned}
x_{k+1} & =A x_{k}+B u_{k}+E d_{k}+G w_{k} \\
y_{k} & =C x_{k}+v_{k}
\end{aligned}\right.
$$

where $d_{k}$ is the unknown input at time $k$ and $E$ is the unknown input gain matrix.

The following assumption is made:

$$
d_{k+1}=d_{k}+\delta_{k}
$$

where $\delta_{k}$ is a random noise. In this way, time-varying unknown input can be taken into account.

An augmented system can be written as follows:

$$
\left\{\begin{aligned}
x_{k+1}^{\prime} & =A_{a} x_{k}^{\prime}+B_{a} u_{k}+G_{a} w_{k}^{\prime} \\
y_{k} & =C_{a} x_{k}^{\prime}+v_{k}
\end{aligned}\right.
$$

with

$$
\begin{gathered}
x_{k}^{\prime}=\left[\begin{array}{ll}
x_{k}^{T} & d_{k}^{T}
\end{array}\right]^{T}, \quad w_{k}^{\prime}=\left[\begin{array}{cc}
w_{k}^{T} & \delta_{k}^{T}
\end{array}\right]^{T} \\
A_{a}=\left[\begin{array}{cc}
A & E \\
0 & I
\end{array}\right], \quad B_{a}=\left[\begin{array}{c}
B \\
0
\end{array}\right] \\
C_{a}=\left[\begin{array}{ll}
C & 0
\end{array}\right] \quad \text { and } \quad G_{a}=\left[\begin{array}{cc}
G & 0 \\
0 & I
\end{array}\right]
\end{gathered}
$$

The augmented state estimate $\hat{x}_{k-m}^{\prime}$ can be carried out as in the previous case:

$$
\hat{x}_{k-m}^{\prime}=\left(P_{m, a}^{T} P_{m, a}\right)^{-1} P_{m, a}^{T}\left(Y_{k}-B_{m, a} U_{k}\right)
$$

where the matrix $P_{m, a}$ and $B_{m, a}$ are built as the matrix $P_{m}$ (5) and $B_{m}(6)$, by replacing matrices $A$, $B$ and $C$ respectively by $A_{a}, B_{a}$ and $C_{a}$.

As previously, expression of the augmented state estimate at time $k$ is deduced from (15):

$$
\hat{x}_{k}^{\prime}=A_{a}^{m} \hat{x}_{k-m}^{\prime}+T_{m, a} U_{k}
$$

with

$$
T_{m, a}=\left[\begin{array}{lllll}
\left(A_{a}^{m-1} B_{a}\right)^{T} & \left(A_{a}^{m-2} B_{a}\right)^{T} & \ldots & B_{a}^{T} & 0
\end{array}\right]^{T}
$$

This formulation allows one to simultaneously obtain state and unknown input estimates of the system.

Example 1: Consider the following unknown input system:

$$
\begin{aligned}
x_{k+1} & =\left[\begin{array}{cc}
0.45 & 0 \\
0 & 0.4
\end{array}\right] x_{k}+\left[\begin{array}{l}
0.1815 \\
1.7902
\end{array}\right] u_{k} \\
& +\left[\begin{array}{c}
0.0129 \\
-1.2504
\end{array}\right] d_{k}+\left[\begin{array}{c}
1 \\
10
\end{array}\right] w_{k} \\
y_{k} & =\left[\begin{array}{ll}
1 & 0 \\
0 & 1
\end{array}\right] x_{k}+v_{k}
\end{aligned}
$$

First a constant unknown input ( $\delta_{k}$ is centered) that occurs in a specified time interval is considered. Afterwards, we consider an unknown input with a ramp

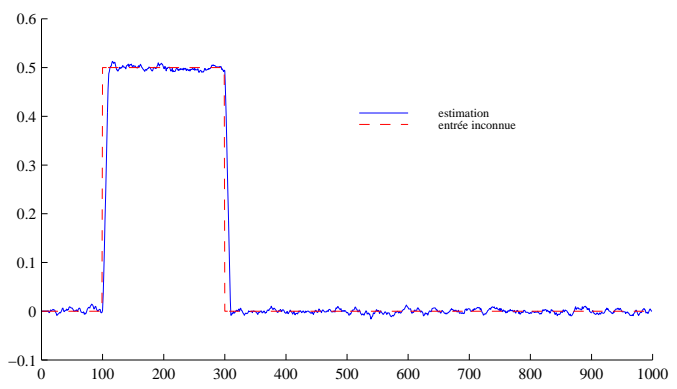

Figure 1. Constant unknown input estimation

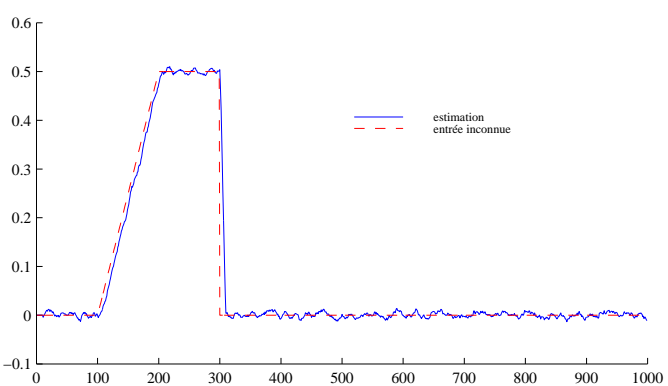

Figure 2. Variable unknown input estimation shape (the mean value of $\delta_{k}$ is different from zero).

Examination of figure 1 allows us to note the good estimation of the unknown input, in spite of a certain delay due to the horizon of observation of the OMF (chosen here equal to $11(m=10))$. Figure 2 presents similar results when the unknown input evolves according to a ramp.

\section{FMO FOR SWITCHING SYSTEMS}

In this section, we consider a system represented by a set of models $M_{i}, i=1, \ldots, r$; each model representing a particular behaviour of the system. The objective is to detect, at each moment, the active model and simultaneously to estimate the state of the system. The transitions from a model to another one are assumed to be described by a Markovien process governed by the a priori known Markov transition matrix $\Pi$ given by:

$$
\Pi=\left[\begin{array}{ccc}
p_{11} & \cdots & p_{1 r} \\
\vdots & \ddots & \vdots \\
p_{r 1} & \cdots & p_{r r}
\end{array}\right]
$$

where $p_{i j}$ is the mode transition probability from the model $M_{i}$ to the model $M_{j}$; we note $\mu_{j}^{k}$ the probability that the $j^{t h}$ model is active at time $k$.

\subsection{Development of the method}

Consider the $j^{\text {th }}$ model described by:

$$
M_{j}:\left\{\begin{aligned}
x_{k+1} & =A_{j} x_{k}+B_{j} u_{k}+G_{j} w_{k} \\
y_{k} & =C_{j} x_{k}+v_{k}
\end{aligned}\right.
$$


The state estimation of this model is carried out using the FMO described in section 2 to give the following equations:

$$
\hat{x}_{k-m}^{j}=\left(P_{j, m}^{T} P_{j, m}\right)^{-1} P_{j, m}^{T}\left(Y_{k}-B_{j, m} U_{k}\right)
$$

and

$$
\hat{x}_{k}^{j}=A_{j}^{m} \hat{x}_{k-m}^{j}+T_{j, m} U_{k}
$$

where the matrices $P_{j, m}, B_{j, m}$ and $T_{j, m}$ are built using the definitions (5), (6) and (10) replacing matrices $A, B$ and $C$ by matrices $A_{j}, B_{j}$ and $C_{j}$ related to the $j^{\text {th }}$ model.

The state estimate $\hat{x}_{k}$ of the switching system is then computed as a weighted sum of the states of the "local" models:

$$
\hat{x}_{k}=\sum_{j=1}^{r} \hat{x}_{k}^{j} \mu_{k}^{j}
$$

Following the work of Bar-Shalom (Bar-Shalom, 1990), the probability that model $j$ is in effect at time $k$ is computed in the following way:

$$
\mu_{k}^{j}=P\left\{M_{j}(k) \mid Y_{k}\right\}
$$

Define $\tilde{Y}_{k-1}$, the observation vector carried out on the horizon $[k-m, k-1]$; we have:

$$
Y_{k}=\left[\begin{array}{ll}
\tilde{Y}_{k-1}^{T} & y_{k}^{T}
\end{array}\right]
$$

Equation (22) can then be written as:

$$
\mu_{k}^{j}=P\left\{M_{j}(k) \mid \tilde{Y}_{k-1}, y_{k}\right\}
$$

Using the Bayes formula, this probability can be transformed into:

$$
\mu_{k}^{j}=\frac{p\left[y_{k} \mid M_{j}(k), \tilde{Y}_{k-1}\right] P\left\{M_{j}(k) \mid \tilde{Y}_{k-1}\right\}}{\sum_{l=1}^{r} p\left[y_{k} \mid M_{l}(k), \tilde{Y}_{k-1}\right] P\left\{M_{l}(k) \mid \tilde{Y}_{k-1}\right\}}
$$

In order to alleviate the notations, let us introduce:

$$
L_{i}(k)=p\left[y_{k} \mid M_{i}(k), \tilde{Y}_{k-1}\right]
$$

Using the total probability theorem, the activation probability of the model $j$ at time $k$, according to the active model at the time $k-1$ can be written as:

$$
\begin{aligned}
& P\left\{M_{j}(k) \mid \tilde{Y}_{k-1}\right\}= \\
& \sum_{i=1}^{r} P\left\{M_{j}(k) \mid M_{i}(k-1), \tilde{Y}_{k-1}\right\} P\left\{M_{i}(k-1) \mid \tilde{Y}_{k-1}\right\}
\end{aligned}
$$

To obtain a recurrence on the computation of the $\mu_{k}^{j}$, we define the following approximation:

$$
P\left\{M_{i}(k-1) \mid \tilde{Y}_{k-1}\right\} \approx P\left\{M_{i}(k-1) \mid Y_{k-1}\right\}=\mu_{k-1}^{i}
$$

Which means to consider that the information given by the first vector of observation $y_{k-m-1}$ of the vector $Y_{k-1}$ defined on the horizon $[k-m-1, k-1]$ is not very important and can be neglected (which depends obviously on the selected horizon). In this case, considering equations (25) to (28) and noticing that, by definition, $P\left\{M_{j}(k) \mid M_{i}(k-1), \tilde{Y}_{k}\right\}=p_{i j}$, the following recurrence on the probability that the system operates according to the model $j$ at the moment $k$ can be established:

$$
\mu_{k}^{j}=\frac{L_{j}(k) \sum_{i=1}^{r} p_{i j} \mu_{k-1}^{j}}{\sum_{l=1}^{r} L_{l}(k) \sum_{i=1}^{r} p_{i l} \mu_{k-1}^{j}}
$$

\subsection{Fault models}

An actuator fault can be modelled by "modifying" the appropriate column of the control input matrix $B$. Thus, a fault on the $i^{\text {th }}$ actuator is described by writing the following equation:

$$
x_{k+1}=A x_{k}+\left(B+\Delta B_{i}\right) u_{k}+w_{k}
$$

where $\Delta B_{i}$ is a matrix of same dimension of $B$; all of its columns are null except the $i^{t h}$ which characterizes the fault on the $i^{t h}$ actuator.

On a same way, a sensor fault is described by:

$$
y_{k}=\left(C+\Delta C_{i}\right) x_{k}+v_{k}
$$

where $\Delta C_{i}$ and $C$ have the same dimension; all of its columns are null except the $i^{t h}$ whicn characterizes the fault on the $i^{\text {th }}$ sensor.

\subsection{An application example}

For the application of the suggested method, we consider a model of normal operating $\left(A_{1}, B_{1}, C_{1}\right)$, a model of fault actuator $\left(A_{2}, B_{2}, C_{2}\right)$ and a model of sensor faults $\left(A_{3}, B_{3}, C_{3}\right)$, with the various matrices being defined by:

$$
\begin{gathered}
A_{i}=\left[\begin{array}{cc}
0.45 & 0 \\
0 & 0.4
\end{array}\right], i=1 \ldots 3 \\
B_{1}=\left[\begin{array}{ll}
0.1815 & 1.7902
\end{array}\right]^{T}, C_{1}=\left[\begin{array}{ll}
1 & 0 \\
0 & 1
\end{array}\right], \\
B_{2}=\left[\begin{array}{ll}
1.1815 & 1.7902
\end{array}\right]^{T}, C_{2}=\left[\begin{array}{ll}
1 & 0 \\
0 & 1
\end{array}\right], \\
B_{3}=\left[\begin{array}{ll}
0.1815 & 1.7902
\end{array}\right]^{T}, C_{3}=\left[\begin{array}{cc}
1.5 & 0 \\
0 & 1.5
\end{array}\right] .
\end{gathered}
$$

To test the method, the following scenario was established: initially the system normally operates, then at time 100, an actuator fault occurs, at time 500, the system returns to the normal operating mode and, at time 800 , sensor faults are introduced.

The results are presented at the figures 3, 4 and 5 where the changes of mode clearly appear; the mode probabilities of the corresponding models, in 


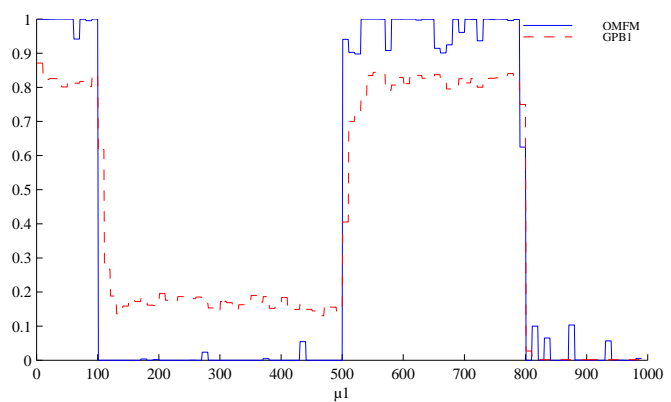

Figure 3. Activation probability of model 1

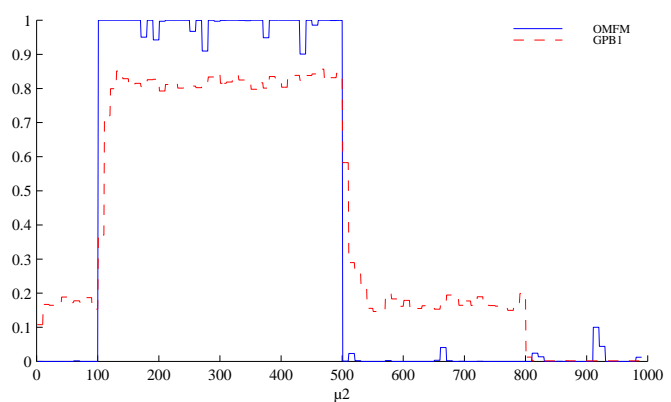

Figure 4. Activation probability of model 2

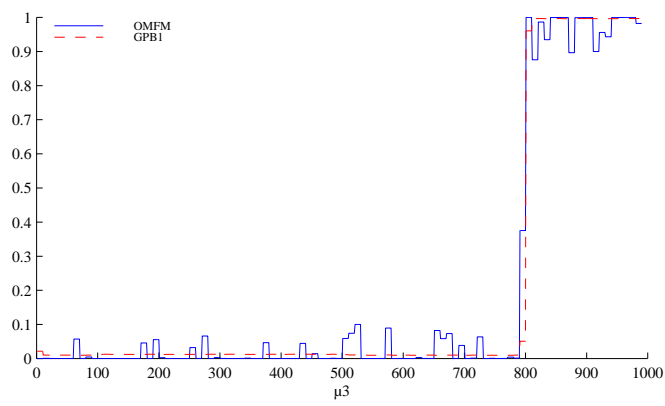

Figure 5. Activation probability of model 3

their respective operation domains, fluctuate around one and thus a detection of the fault is carried out. Clearly, the results of the suggested method are better than those of GPB1 method. This fact can be explained by the sensitivity of GPB1 to the noise affecting the system state (the signal to noise ratio in the examples is equal to $10 \%$ ). This, allows us to conclude that the use of a finite memory observer, for fault detection in switching systems, is less sensitive to noise than traditional GPB methods.

\section{EXTENSION OF THE METHOD TO UNKNOWN INPUT SYSTEM}

The suggested method described in the previous section can be applied to unknown input systems. In this context, the finite memory observer of the second section is replaced by a finite memory observer with unknown input (see section 3 ).

The $j^{t h}$ model is written as follows:

$$
M_{j}\left\{\begin{aligned}
x_{k+1} & =A_{j} x_{k}+B_{j} u_{k}+E d_{k}+G w_{k} \\
y_{k} & =C_{j} x_{k}+v_{k}
\end{aligned}\right.
$$

where $d_{k}$ is the unknown input at the time $k$ and $E$ is the unknown input gain matrix.

Using an augmented model for each model $M_{j}$, as mentioned in section 3, the state and the unknown input can be simultaneously estimated. The estimation of the system state is then obtained following the steps given in subsection 4.1 .

Example 2: We consider the same models of normal operation, actuator fault and sensor faults as described previously, but under the effect of the unknown input $d_{k}$ whose influence matrix is:

$$
E=[0.0129-1.2504]^{T}
$$

In order to test the method, the following scenario was established: initially the system normally operates, at time 100 an unknown input occurs with a constant magnitude, then at time 200, an actuator fault occurs, at time 300, the unknown input becomes null, at time 500, the system returns to the normal operating mode and, at time 800 , sensor faults are introduced.

Clearly the results, in the presence of noise, are shown on figures 6 to 8 , highlights the changes from one mode to another, allowing the detection of faults. The figure 9 shows the estimation of the unknown input in the absence of noise. However, in the presence of noise (the signal to noise ratio is equal to $10 \%$ ), this estimate is given in figure 10 . We can claim that the use of a finite memory observer with unknown input in switched system, for fault detection and unknown input estimation, gives good results in spite of the presence of noise.

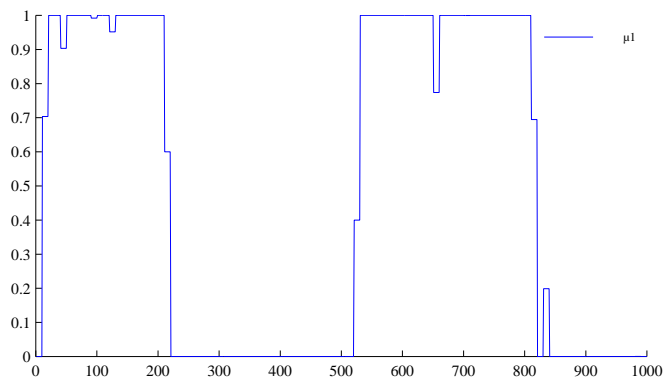

Figure 6. Activation probability of model 1 in the presence of noise

\section{CONCLUSION}

In this work, the structure of a Finite Memory Observer is proposed to handel the FDI issue under the eventual presence of unknown input. This kind of 


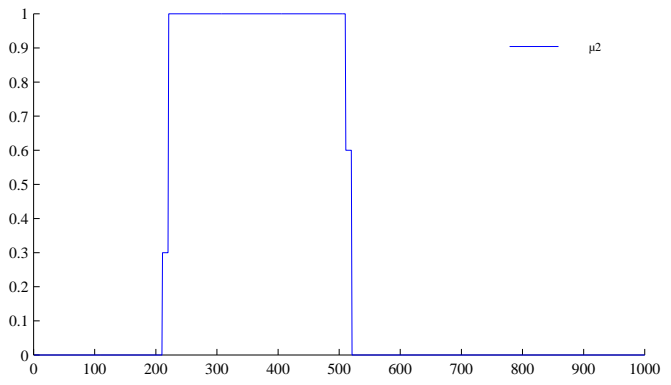

Figure 7. Activation probability of model 2 in the presence of noise

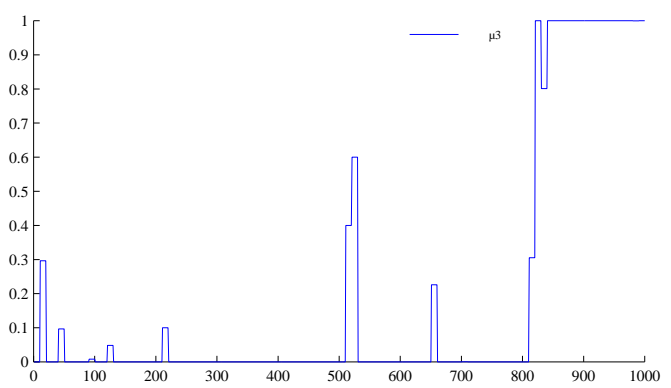

Figure 8. Activation probability of model 3 in the presence of noise

observer was applied within the framework of Markovian switching systems for which the switching event between models must be detected. Comparison of the obtained results with those of GPB approach was carried out on a computer simulation example. The use of a finite memory observer, based on measured outputs only, in contrast to the GPB1 method which uses estimates, gives better results, especially in the presence of noises.

Finally, the suggested method was extended to the case of systems subjected to unknown input. In this situation, the detection of the moments of commutation is performed with the estimation of the unknown input.

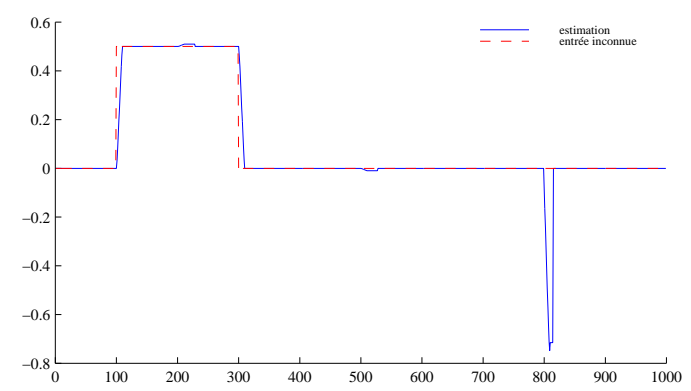

Figure 9. Unknown input estimation in the absence of noise

\section{REFERENCES}

Bar-Shalom, Y. (1990). Multitarget-multisensor tracking: advanced applications. Artech House.

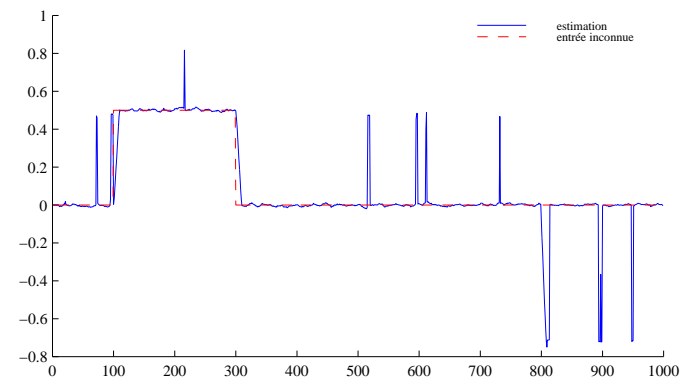

Figure 10. Unknown input estimation in the presence of noise

Bar-Shalom, Y. and X. Li (1993). Estimation and tracking: principles, technique and software. Artech House.

Bar-Shalom, Y., K.C. Chang and H.A. Blom (1989). Tracking a manoeuvering target using input estimation versus interacting multiple model algorithm. IEEE Transactions on Aerospace and Electronic Systems 25, 296-300.

Blanke, M., M. Kinnaert, J. Lunze and M. Staroswiecki (2003). Diagnosis and fault-tolerant control. Springer.

Chow, E. and A. S. Willsky (1984). Analytical redundancy and the design of robust failure detection systems.. IEEE Transactions on Automatic control 29, 603-614.

Gasso, K., G. Mourot and J. Ragot (2001). Structure identification in multiple model representation: elimination and merging of local models. 40th Conference on Decision and Control, Orlando.

Hanlon, P.D. and P.S. Maybeck (1998). Interrelationship of single-filter and multiple-model adaptative algorithms. IEEE Transactions on Aerospace and Electronic Systems 34, 934-946.

Kratz, F., S. Bousghiri and G. Mourot (1994). A finite memory observer approach to the design of fault detection algorithms. American Control Conference, Baltimore pp. 3574-3576.

Medvedev, A. (1996). State estimation and fault detection by a bank of continuous finite-memory filters. IFAC World Congress, San Francisco, USA D, 177-182.

Murray-Smith, R. and T.A. Johansen (1997). Multiple Model Approaches to Modelling and Control. Taylor and Francis. UK.

Nuninger, W., F. Kratz and J. Ragot (1998). Finite memory generalised state observer approach for failure detection in dynamic systems. 37th IEEE Conference on Decision and Control, Tampa, Florida, USA pp. 581-585.

Patton, R., P.M. Frank and R. Clark (1989). Fault diagnosis in dynamic systems. Prentice Hall. 